\title{
Cooperative Target Defense Differential Game with a Constrained-maneuverable Defender
}

\author{
David W. Casbeer, Eloy Garcia, Zachariah E. Fuchs, and Meir Pachter
}

\begin{abstract}
This paper addresses the active target defense differential game where a Target aircraft is pursued by an Attacker missile, and a Defender missile is employed in order to intercept the Attacker and protect the evading Target. This paper extends the results concerning the active target defense differential game by allowing for the Defender's turning rate to be constrained. The restriction imposed on the Defender's turning rate is of great operational relevance since initially the Defender's heading might be different than the otherwise optimal heading when simple motion is assumed.
\end{abstract}

\section{INTRODUCTION}

Multi-agent pursuit-evasion scenarios present important and challenging problems in aerospace control. One or more pursuers try to maneuver to close in on one or more evaders, who, simultaneously, are striving to escape the pursuers. Several types of pursuit-evasion scenarios involving many agents have been studied. This problem is naturally posed as a differential game [1], [2].

In this paper we consider a zero-sum, three-agent, pursuitevasion differential game. A two-agent team is formed which consists of a Target $(T)$ and a Defender $(D)$; the Attacker $(A)$ is the opposition. The Attacker's objective is to capture the Target, while the Target tries to evade the Attacker. The Defender pursues the Attacker to assist the Target. Cooperation between the Target and the Defender is such that the Defender will capture the Attacker before the latter reaches the Target.

Such a scenario of active target defense has been analyzed in the context of cooperative optimal control in [3], [4]. More recent work has investigated different guidance laws for the agents $A$ and $D$. In [5] the authors addressed the case where the Defender implements Command to the Line of Sight (CLOS) guidance to pursue the Attacker. A different guidance law was given by Yamasaki et.al. [6], [7]. These authors investigated an interception method called Triangle Guidance (TG), which experimentally was shown to outperform Proportional Navigation (PN) guidance laws. In all these approaches, the level of cooperation between $T$ and $D$ was constrained by implementing Defender guidance laws without regard to the Target's trajectory.

Different types of cooperation have been recently proposed in [8], [9], [10], [11], [12] for the target, attacker, defender

D. Casbeer is with the Control Science Center of Excellence, AFRL, WPAFB, OH. david.casbeer@us.af.mil

E. Garcia is a contractor (Infoscitex Corp.) with Control Science Center of Excellence, AFRL, WPAFB, OH. elgarcialinfoscitex.com

Z. Fuchs is with the Dept of Elec Eng, Wright State Univ., OH. zachariah. fuchsewright. edu

M. Pachter is with the Dept of Elec Eng, Air Force Institute of Technology, WPAFB, OH. meir.pachtereafit.edu
(TAD) scenario. In these papers the Target represents an aircraft trying to evade a missile homing on it. The Defender is a missile launched by the aircraft (or a wingman) in order to intercept and destroy the Attacker in order to guarantee the survival of the aircraft. Thus, in [9] optimal policies (lateral acceleration for each agent including the Attacker) are provided for the case of an aggressive Defender, that is, the Defender has a definite maneuverability advantage. A linear quadratic optimization problem is posed where the Defender's control effort weight is driven to zero to increase its aggressiveness. The work [10] provided a game theoretical analysis of the TAD problem using different guidance laws for both the Attacker and the Defender. The cooperative strategies in [11] allow for a maneuverability disadvantage for the Defender with respect to the Attacker and the results show that the optimal Target maneuver is either constant or arbitrary. Shaferman and Shima [12] implemented a Multiple Model Adaptive Estimator (MMAE) to identify the guidance law and parameters of the incoming missile and optimize a Defender strategy to minimize its control effort.

Our preliminary work involving the differential game formulation of the cooperative target defense problem assumed that all agents had simple motion ([13], [14], [15]). This paper extends these previous results to consider the practically important case where the Defender's turning rate is bounded, and, therefore, it is not able to achieve its optimal heading instantaneously.

The paper is organized as follows. Section II describes the engagement scenario. Section III provides an interception strategy for the Defender to intercept the Attacker despite its turning rate constraint. Section IV provides the main result of the paper, namely, the optimal strategies for each one of the three agents which are given by the solution of the differential game discussed in the paper. Illustrative examples are given in Section V, and concluding remarks are made in Section VI.

\section{Problem Statement}

In this section, we formally describe the pursuit and evasion differential game addressed in this paper. We consider the TAD scenario where the Attacker and the Defender missiles have the same speeds. We assume that the Attacker and the Target can turn infinitely fast (or that their initial headings are close to optimal, and any small adjustments are negligible). The justification for this assumption is as follows. First, the Attacker has been launched and is on a course that is close to the optimal. Second, the Target, aware of the attack, has already started veering away from the 
Attacker, thus its heading is close to optimal. The Defender, on the other hand, reacting late to the situation, may have an initial heading, denoted as $\psi_{0}$, much different than its optimal heading, and it needs to perform a hard turn-straight maneuver in order to intercept the Attacker. Thus, let $r$ be the minimal radius of the Defender's turning circle, whereas the Target and the Attacker have simple motion.

The dynamics of the three agents in the realistic plane are given by:

$$
\begin{array}{ll}
\dot{x}_{T}=V_{T} \cos \hat{\phi}, & \dot{y}_{T}=V_{T} \sin \hat{\phi} \\
\dot{x}_{A}=V_{A} \cos \hat{\chi}, & \dot{y}_{A}=V_{A} \sin \hat{\chi} \\
\dot{x}_{D}=V_{D} \cos \hat{\psi}, & \dot{y}_{D}=V_{D} \sin \hat{\psi}
\end{array}
$$

such that

$$
\dot{\hat{\psi}} \in\left[-\Omega_{M}, \Omega_{M}\right]
$$

where $\hat{\phi}, \hat{\chi}$, and $\hat{\psi}$ represent the instantaneous headings of the Target, the Attacker, and the Defender, respectively. The speeds of the Target, Attacker, and Defender are denoted by $V_{T}, V_{A}$, and $V_{D}$, respectively, which are assumed to be constant. Additionally, $\Omega_{M}=V_{D} / r$.

Define the speed ratio parameter $\alpha=V_{T} / V_{A}$. In general, we have that the Attacker missile is faster than the Target aircraft, so that $\alpha<1$. In this work we assume the Attacker and Defender missiles are in the same class, so $V_{D}=V_{A}$. Thus, without loss of generality, we address the active target defense differential game using the normalized (by $V_{A}$ ) speeds: $V_{A}=V_{D}=1$ and $V_{T}=\alpha$. In this game the Attacker pursues the Target and tries to capture it. The Target and the Defender cooperate in order for the Defender to intercept the Attacker before the latter captures the Target. Thus, the Target-Defender team search for an optimal strategy to maximize the distance between the Target and the Attacker at Defender-Attacker interception time. The Attacker will search for its corresponding optimal strategy in order to minimize the terminal $A-T$ separation.

\section{INTERCEPTION OF THE ATTACKER BY THE DEFENDER}

In this section, we address the Defender's strategy that leads to interception of an Attacker with constant heading, without regard to the Target. As will be shown later, a constant heading is the optimal Attacker strategy. Section IV addresses the active target defense differential game and the optimal strategies to $\min / \max$ the final separation between the Target and the Attacker.

When the Attacker chooses a constant heading $\hat{\chi}$, the Defender will implement, in general, a hard turn-straight strategy in order to intercept the Attacker. Depending on its initial heading, the Defender turning left or right could be beneficial or detrimental. This ambiguity is dealt with by solving for both possibilities; that is, when the Attacker chooses some constant heading $\hat{\chi}$ the Defender evaluates the solution of two similar control problems and opts for the solution that maximizes his payoff.
In order to find the interception strategy, we consider the X-Y rotating reference frame centered at the Attacker's position, where the $\mathrm{X}$-axis is defined by the line passing through $A$ and the center of the Defender's left turning circle, denoted by $C_{l}$ (or correspondingly, to the center of the Defender's right turning circle, $C_{r}$ ). The Y-axis is then the orthogonal line passing through the point $A=(0,0)$. Since both cases can be treated in a similar way, we focus on the case of determining the interception point when turning left. The coordinates of the center of the circle are given by $C_{l}=\left(x_{l}, 0\right)$. The coordinates of the Target and the Defender in this frame are given by $T=\left(x_{T}, y_{T}\right)$ and $D=\left(x_{D}, y_{D}\right)$. Heading angles measured in these rotating frames will not have the "^" in order to differentiate from the fixed frame.

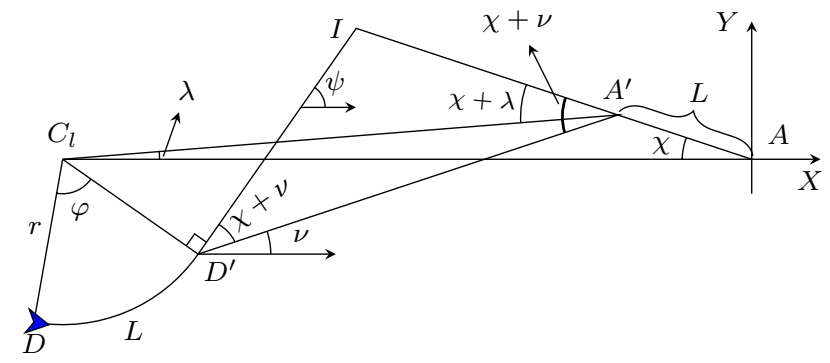

Fig. 1: Determination of $L(\chi)$

Proposition 1: Let the Defender's initial heading be $\psi_{0}$. For an Attacker's strategy $\chi$, such that the Defender is able to intercept the Attacker by following the strategy turn-straight, the length, $L=r \varphi$, of the circular arc traveled by the Defender before going on a straight line is found by solving the following equation:

$$
\Lambda(\chi, L)=0
$$

where

$\Lambda(\chi, L)=\arctan \left(\frac{L \sin \chi+r \cos \chi}{-x_{l}-L \cos \chi+r \sin \chi}\right)-\frac{\Omega_{M} L+\psi_{0}-\chi}{2}$.

Proof: Using the geometry and angles defined in Fig. 1, the locus of points where the Defender intercepts the Attacker can be characterized as follows. Since both the Defender and the Attacker have the same speed, the total distance traveled by the Defender and Attacker must be equal, i.e., $L+\overline{D^{\prime} I}=\overline{A I}$, where $\overline{A I}$ indicates the length of the segment from point $A$ to point $I$. Given the Defender's initial heading $\psi_{0}$, the Defender's heading at the end of the turn (and consequently its heading while traveling along a straight line) is given by $\psi=\psi_{0}+\Omega_{M} L$. When the Defender finishes turning and it is located at point $D^{\prime}$ the Attacker is located at point $A^{\prime}=\left(x_{A}^{\prime}, y_{A}^{\prime}\right)$, where $x_{A}^{\prime}=-L \cos \chi$ and $y_{A}^{\prime}=L \sin \chi$. Thus, the following holds

$$
\frac{\overline{C_{l} A^{\prime}}}{\sin (\pi / 2+\chi+\nu)}=\frac{r}{\sin (\nu-\lambda)},
$$

where $\lambda$ represents the LOS (line of sight) angle from $C_{l}$ to $A^{\prime}$. We can write the following:

$$
\begin{aligned}
& \overline{C_{l} A^{\prime}}(\sin \nu \cos \lambda-\cos \nu \sin \lambda) \\
& \quad=r(\sin (\pi / 2+\chi) \cos \nu+\cos (\pi / 2+\chi) \sin \nu)
\end{aligned}
$$



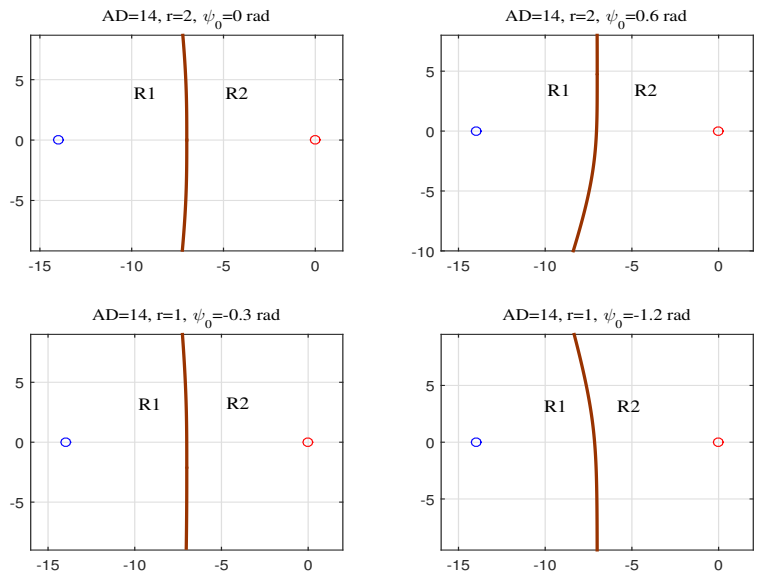

Fig. 2: Examples of the interception curve. ○:Attacker; $\circ:$ Defender

and by substituting $\cos \lambda=\frac{x_{A}^{\prime}-x_{l}}{\overline{C_{l} A^{\prime}}}$ and $\sin \lambda=\frac{y_{A}^{\prime}}{\overline{C_{l} A^{\prime}}}$ in (6) we can write the following equation:

$$
\frac{\sin \nu}{\cos \nu}=\frac{L \sin \chi+r \cos \chi}{-x_{l}-L \cos \chi+r \sin \chi} .
$$

Also note that $\psi_{0}+\Omega_{M} L=\chi+2 \nu$, and we can write (7) as shown in (3).

Given the Defender turning constraints, the Defender will intercept the Attacker at some point $I=\left(x_{I}, y_{I}\right)$ that lies on the curve parameterized by (3). Given an initial heading $\psi_{0}$, we say that the arc length $L$ is feasible (and so is its corresponding turn angle $\varphi=L / r$ ), if there exists a heading $\chi$ such that $D$ intercepts $A$. Since, there exist multiple feasible values of $L$ for a given heading $\chi$, the following definition is needed.

Definition 1: Assume that the set of feasible interception strategies $L$ is not empty for a given $\chi$. The optimal interception strategy $L^{*}$ given $\psi_{0}$ and $\chi$ is defined as follows

$$
\begin{aligned}
L^{*}= & \arg \min _{L} d_{A} \\
& \text { subject to } \Lambda(\chi, L)=0,
\end{aligned}
$$

where

$$
d_{A}=\frac{r^{2}+L^{2}-x_{l}^{2}}{L+x_{l} \cos \chi}=\overline{A I} .
$$

Corresponding to the optimal interception strategy let us define

$$
\Lambda\left(\chi, L^{*}\right)=0
$$

as the constraint (3) when using the optimal arc length $L^{*}$. Fig. 2 shows a few examples of the curve (11). In Fig. 2.a the separation between the Attacker and the Defender is $\overline{A D}=$ 14 , the radius of the maximum turning rate circle is $r=$ 2 , and the initial Defender heading is $\psi_{0}=0 \mathrm{rad}$. The remaining plots show similar curves for different values of the same parameters. Note that the curvature of (11) mainly depends on the ratio $x_{l} / r$.

Notice that the curve (11) divides the X-Y plane into two regions. All points in Region 1 can be reached by the
Defender before the Attacker by using the 'Turn-Straight' strategy. Similarly, all points in Region 2 can be reached by the Attacker before the Defender if the Attacker keeps a constant heading, that is, if it travels in a straight line. Here, we assume that the ratio $x_{l} / r$ is sufficiently large such that the Attacker never enters the Defender's turning circle. Also, recall that the Attacker's objective is to get as close to the Target as possible when the Defender reaches the Attacker.

\section{Optimal Strategies}

\section{A. The differential game}

We assume that the Target is initially positioned in Region 1, which is delineated by the curve (11). In this case, the Target chooses point $v$ on that curve in order to run away from that point, and the Attacker chooses his aimpoint $u$ on the same curve. Additionally, the Defender tries to intercept the Attacker by choosing his aimpoint $w$, also on the curve (11). The Target, the Defender, and the Attacker are faced with the following minmax optimization problem: $\min _{u} \max _{v, w} J(u, v, w)$, where $J(u, v, w)$ is the distance between the Target terminal position $T^{\prime}$ and the point on the curve where the Attacker is intercepted by the Defender.

The Defender helps the Target to escape by intercepting the Attacker at the point $u$. Therefore, the Defender's optimal policy is $w^{*}(u, v)=u$ in order to guarantee interception of the Attacker. The point $u$ is determined by the choice of the Attacker heading $\chi$. Given the Attacker's heading, the Defender is able to determine the length $L$ of its corresponding circular arc path by solving eq. (11).

Since the Defender's optimal policy is $w^{*}=u$, the decision variables $u$ and $v$ jointly determine $J(u, v)$, where $J(u, v)$ is the distance between the Target terminal position $T^{\prime}$ and the point $I=u$ on the curve (11) where the Attacker is intercepted by the Defender.

Proposition 2: Assume that the Target is initially positioned in Region 1 of the plane divided by the curve (11). Given the cost/payoff function $J(u, v)$, the solution $u^{*}$ and $v^{*}$ of the optimization problem $\min _{u} \max _{v} J(u, v)$ is such that

$$
u^{*}=v^{*} \text {. }
$$

Moreover, the Target's strategy is $v^{*}(u)=$ $\arg \max _{v} J(u, v)=u$ so that it suffices to solve the optimization problem

$$
\begin{aligned}
& \min _{\chi} J(\chi, L) \\
& \text { subject to } \Lambda(\chi, L)=0,
\end{aligned}
$$

where

$J(\chi, L)=\alpha \sqrt{x_{I}^{2}+y_{I}^{2}}+\sqrt{\left(x_{I}-x_{T}\right)^{2}+\left(y_{I}-y_{T}\right)^{2}}$,

and $x_{I}(\chi, L), y_{I}(\chi, L)$ are the coordinates of the interception point $I=u$.

The active target defense differential game where all agents are described by simple motion models, that is, turning rate constraints are not incorporated into the Defender's dynamics, was addressed in [13]. The differential game in 
[13] was analyzed by attaching a rotating Cartesian frame in such a way that the extension to infinity of $\overline{A D}$ in both directions represents the $\mathrm{X}$-axis and the orthogonal bisector line of $\overline{A D}$ represents the Y-axis. The solution of the differential game is obtained by solving the quartic equation in $y(\geq 0)$

$$
\begin{aligned}
& \left(1-\alpha^{2}\right) y^{4}-2\left(1-\alpha^{2}\right) \bar{y}_{T} y^{3} \\
& +\left(\left(1-\alpha^{2}\right) \bar{y}_{T}^{2}+\bar{x}_{A}^{2}-\alpha^{2} \bar{x}_{T}^{2}\right) y^{2} \\
& -2 \bar{x}_{A}^{2} \bar{y}_{T} y+\bar{x}_{A}^{2} \bar{y}_{T}^{2}=0,
\end{aligned}
$$

where $\bar{x}_{A}$ represents the coordinate of the Attacker in the relative Cartesian frame (note that $\left.\bar{y}_{A}=0\right)$ and $\left(\bar{x}_{T}, \bar{y}_{T}\right)$ represent the coordinates of the Target in the same frame.

Now, when explicitly considering the Defender's turning rate constraint, we have a point $D_{0}(\varphi)$ on the circle involute, see Fig. 3, that defines an equivalent problem with constant heading from this initial position $D_{0}(\varphi)$. This is true because the heading at the point $D^{\prime}$ is the same, no matter if the Defender traveled along the turning circle from $D$ or moved straight from $D_{0}$. Thus, the choice of $D_{0}$ directly determines the distance $Y_{I}(\varphi)$ of the interception point $I=\left(0, Y_{I}\right)$ in the reduced state space $(\overline{\mathrm{X}}, \overline{\mathrm{Y}})$ which is based on $D_{0}$ and $A$ as shown in Fig. 3. In this reduced state space the coordinates of the Attacker are given by $\left(\bar{x}_{A}(\varphi), 0\right)$ and the coordinates of the Target are given by $\left(\bar{x}_{T}(\varphi), \bar{y}_{T}(\varphi)\right)$. This equivalent scenario corresponds to the case in [13] and (14) can be solved to obtain $y(\varphi)=y\left(\bar{x}_{A}(\varphi), \bar{x}_{T}(\varphi), \bar{y}_{T}(\varphi)\right)$.

Proposition 3: The optimal interception strategy $\varphi$ is given by $D_{0}(\varphi)$ such that $Y_{I}(\varphi)=y(\varphi)$.

The complexity of the problem by including Defender turning rate constraints significantly increases compared to the simple motion case in [13]. It can be seen that a direct solution $\varphi$ to the equation $Y_{I}(\varphi)=y(\varphi)$ is difficult to obtain because $y(\varphi)$ is not explicitly given as a function of $\varphi$ but the quartic equation (14) needs to be solved.

To facilitate the analysis, we will write the cost/payoff function (13) in terms of the variable $\varphi$, which is the angle subtended by the arc traveled while the defender is turning. Notice that we can transform the problem by adjusting the initial location as follows. Place the Defender on the circle involute depicted in Fig. 3, with its heading aligned with the tangent to the circle. The position, $D_{0}=(x, y)$, on the circle involute is parameterized using $\varphi$ and is given by

$$
\begin{aligned}
& x=x_{l}+r(\sin (\psi)-\varphi \cos (\psi)) \\
& y=-r(\cos (\psi)+\varphi \sin (\psi))
\end{aligned}
$$

where $\psi=\psi_{0}+\varphi$ [16]. As seen in Fig. 4, an hypothetical Defender at this transformed initial location $D_{0}$ and heading $\psi_{0}+\varphi$ would travel the same distance $L$ and be at the same heading $\psi_{0}+\varphi$ when it arrived at $D^{\prime}$ as the real starting location $D$ with heading $\psi_{0}$.

\section{B. Solution of the differential game}

In order to solve the active target defense differential game with Defender turning rate constraint we will use the rotating Cartesian frame shown in Fig. 4 and equations (15) to write

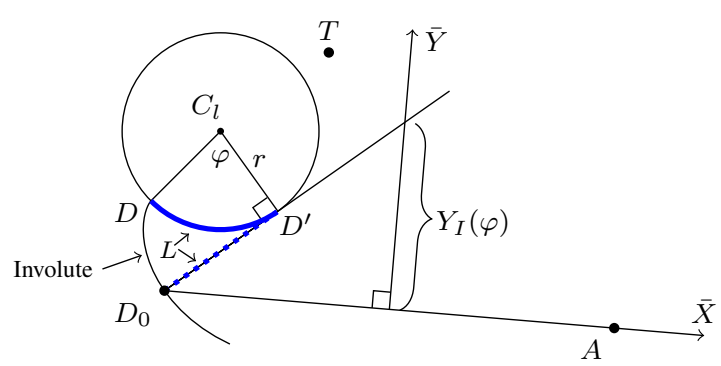

Fig. 3: Circle involute is the locus of equivalent starting points for the Defender.

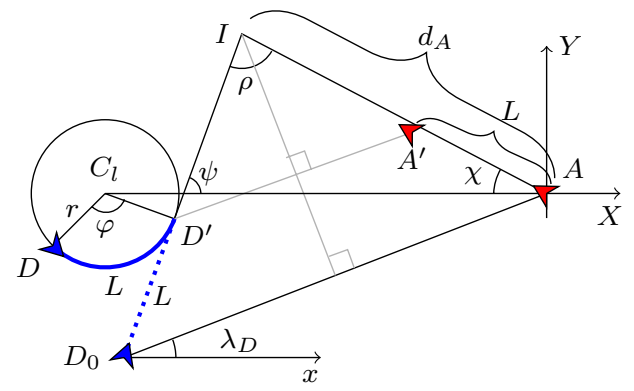

Fig. 4: Equivalent scenario where Defender travels on a straight line

the interception coordinates $I=\left(x_{I}, y_{I}\right)$ and the distance $d_{A}$ between point $A$ and point $I$ only in terms of the variable $\varphi$. In order to do this task we obtain the corresponding Attacker heading $\chi(\varphi)$ such that the Defender and the Attacker meet at point $I$. We will show in Proposition 4 that, once $\varphi^{*}$ is determined, any strategy other than $\chi^{*}\left(\varphi^{*}\right)$ is detrimental to the Attacker.

Theorem 1: Assume that the optimal turning choice is to turn left. Then, the optimal interception point $I$ that minimizes (13) has coordinates $I^{*}=\left(x_{I}\left(\varphi^{*}\right), y_{I}\left(\varphi^{*}\right)\right)$ where $\varphi^{*}$ is the minimum feasible $\varphi$ that solves $F(\varphi)=0$ in the range $0 \leq \varphi<2 \pi$ and minimizes the cost/payoff function

$$
J(\varphi)=\alpha d_{A}(\varphi)+\overline{T I}
$$

where

$$
\begin{array}{r}
F(\varphi) \triangleq\left(b_{l} \sin \psi+1\right)\left(d_{A}+\alpha \overline{T I}\right) \\
-\left(\cos \psi\left(x_{T}-\varphi y_{T}\right)+\sin \psi\left(\varphi x_{T}+y_{T}\right)+b_{l} y_{T}\right), \\
d_{A}(\varphi)=-r \frac{\frac{1}{2}\left(b_{l}^{2}+1+\varphi^{2}\right)+b_{l}(\sin \psi-\varphi \cos \psi)}{b_{l} \cos \psi-\varphi}, \\
\overline{T I}=\sqrt{\left(x_{I}(\varphi)-x_{T}\right)^{2}+\left(y_{I}(\varphi)-y_{T}\right)^{2}}, \\
x_{I}(\varphi)=r \frac{\left.\frac{1}{2}\left(b_{l}^{2}-1+\varphi^{2}\right) \cos \psi-\varphi \sin \psi-b_{l} \varphi\right)}{b_{l} \cos \psi-\varphi}, \\
y_{I}(\varphi)=r \frac{\left.-\frac{1}{2}\left(b_{l}^{2}+1-\varphi^{2}\right) \sin \psi+\varphi \cos \psi-b_{l}\right)}{b_{l} \cos \psi-\varphi}
\end{array}
$$


$b_{l}=x_{l} / r$

Proof. See Appendix A.

In general, we have to look for the best solution in either direction. The optimal turning angle $\varphi^{*}$, such that $0 \geq \varphi>$ $-2 \pi$, when turning right can be found following a similar approach. In this case the cost/payoff function is given by (16) where $d_{A}(\varphi)$ and $\overline{T I}$ are replaced with

$$
\begin{gathered}
d_{A}(\varphi)=-r \frac{\frac{1}{2}\left(b_{r}^{2}+1+\varphi^{2}\right)+b_{r}(\varphi \cos \psi-\sin \psi)}{b_{r} \cos \psi+\varphi}, \\
\overline{T I}=\sqrt{\left(x_{I}(\varphi)-x_{T}^{r}\right)^{2}+\left(y_{I}(\varphi)-y_{T}^{r}\right)^{2}},
\end{gathered}
$$

where $x_{T}^{r}$ and $y_{T}^{r}$ represent the coordinates of the Target in the right turn frame and the interception coordinates are given by

$$
\begin{aligned}
& x_{I}(\varphi)=r \frac{\left.\frac{1}{2}\left(b_{r}^{2}-1+\varphi^{2}\right) \cos \psi-\varphi \sin \psi+b_{r} \varphi\right)}{b_{r} \cos \psi+\varphi}, \\
& y_{I}(\varphi)=r \frac{\left.-\frac{1}{2}\left(b_{r}^{2}+1-\varphi^{2}\right) \sin \psi+\varphi \cos \psi+b_{r}\right)}{b_{r} \cos \psi+\varphi},
\end{aligned}
$$

where $b_{r}=x_{r} / r$. The function $F(\varphi)$ is now given by:

$$
\begin{aligned}
F(\varphi) & \triangleq\left(b_{r} \sin \psi-1\right)\left(d_{A}+\alpha \overline{T I}\right) \\
& +\cos \psi\left(x_{T}-\varphi y_{T}\right)+\sin \psi\left(\varphi x_{T}+y_{T}\right)-b_{r} y_{T}
\end{aligned}
$$

Proposition 4: Saddle point equilibrium. Consider the case where the target is in Region 1 of the plane divided by the curve $\Lambda(\chi, L)=0$. The strategy $\varphi^{*}$ given in Theorem 1 , where the Defender turns, from $\psi_{0}$, to acquire the heading $\varphi^{*}+\psi_{0}$ and the strategy of the Attacker of heading to the point $I^{*}=\left(x_{I}\left(\varphi^{*}\right), y_{I}\left(\varphi^{*}\right)\right)$, which is determined by $\varphi^{*}$, together with the strategy of the Target of running away from the point of interception of the Attacker by the Defender $I^{*}$, constitute a strategic saddle point, that is

$$
\begin{aligned}
& \left\{J\left(u^{*}, v^{*}, w\right), J\left(u^{*}, v, w^{*}\right), J\left(u^{*}, v, w\right)\right\} \\
& <J\left(u^{*}, v^{*}, w^{*}\right)<J\left(u, v^{*}, w^{*}\right) .
\end{aligned}
$$

Proof: The full proof compares of four cases to the optimal: 1) $A$ acts non-optimally, 2) $T$ acts non-optimally, 3) $D$ acts non-optimally, and 4) both $T$ and $D$ act nonoptimally. For the sake of space we only show the proof for case 1 , the proofs for the other three cases follows similar logic.

1) Suppose the Attacker, the minimizer, deviates from his optimal strategy by choosing a different aimpoint $I^{\prime}=$ $\left(x_{I}(\varphi), y_{I}(\varphi)\right)$, where $\varphi \neq \varphi^{*}$. Suppose also that the Target-Defender team acts optimally, that is, the Attacker is intercepted by the Defender at $I^{\prime}$, and the Target runs away from $I^{\prime}(\varphi)$. Under this assumption the cost $J(\varphi)$ increases since $\varphi \neq \varphi^{*}$, that is, $J\left(\varphi^{*}\right)<J(\varphi)$, equivalently, $J\left(u^{*}, v^{*}, w^{*}\right)<J\left(u, v^{*}, w^{*}\right)$. This is detrimental to the Attacker. Note that since the Attacker is choosing $\varphi \neq \varphi^{*}$, the Defender needs to find the corresponding length of its own turn by solving eq. (11). Also note that if the Attacker does not follow a constant heading and its trajectory is not a straight line, then it will not be able to reach the curve

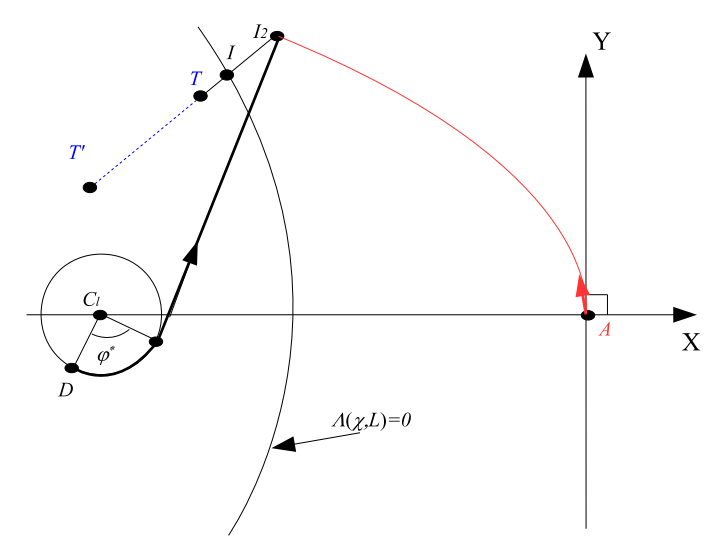

Fig. 5: Non-optimal Attacker with a non-constant heading

$\Lambda(\chi, L)=0$, and it will be intercepted in Region 2 of the corresponding X-Y plane. For any interception point $I_{2}$ in Region 2, there exists a corresponding point $I$ on the curve $\Lambda(\chi, L)=0$, see Fig. 5, such that $\overline{I T}<\overline{I_{2} T}$ and the Target's strategy will be to run away from $I$.

\section{Numerical solution}

The Defender's interception strategy and the optimal angle $\varphi^{*}$ that solves the differential game require the solutions of transcendental equations. Furthermore, the solutions need to belong to a predetermined range. For instance, equation (17) may have more than one solution in the interval $[0,2 \pi)$. However, the range of physically meaningful solutions can be constrained in order to determine a minimum of (16) that provides a feasible interception of the Attacker by the Defender. We first realize that when $\varphi=\varphi_{c}$ both, (16) and (17), tend to infinity. This value of $\varphi$ corresponds to the case where the Defender and the Attacker remain each one on their own starting region and do not cross the curve $\Lambda(\chi, L)=0$.

A simple method to numerically find the root of (17) in the range $\left[0, \varphi_{c}\right)$ is the bisection method (other common methods like Newton's could converge to a solution outside of the interval of interest). This method guarantees convergence to a root of $(17)$ in $\left[0, \varphi_{c}\right)$ if there is one. Another issue is that if (16) has more than one local extremum then there is no guarantee of convergence to the global minimum.

On the other hand the sequential optimization method in [17] and [18] guarantees convergence to the global minimum of (16) within a predetermined range $\left[0, \varphi_{c}\right)$.

Both methods have been implemented in order to determine the solution of the differential game in the following example.

\section{ILLUSTRATIVE EXAMPLES}

Example 1. Consider the following initial positions in the realistic plane: $A=(0,0), D=(-15,0)$, and $T=(-12,8)$. Let the minimum turning radius of $D$ be $r=2$. The initial heading of the Defender in the realistic plane is $\psi_{0}=-0.1$ $\mathrm{rad}$. The optimal turning angle then is $\varphi^{*}=0.939 \mathrm{rad}$ and the optimal trajectories are shown in Fig. 6(a). 


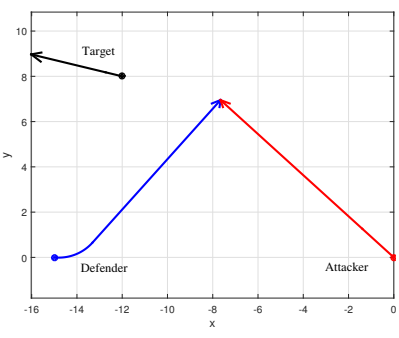

(a)

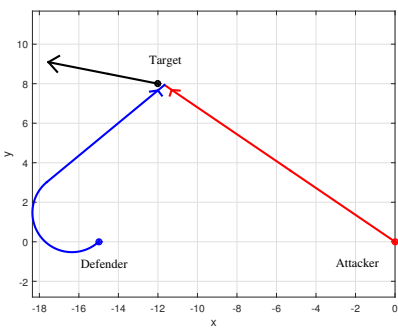

(b)
Fig. 6: Optimal solution for Examples 1 and 2, respectively, in subfigures (a) and (b).

Example 2. Consider the same parameters and initial positions as in Example 1 except that the initial heading of $D$ is $\psi_{0}=-2.4 \mathrm{rad}$. In this case the optimal turning angle is negative and equal to $\varphi^{*}=-3.193 \mathrm{rad}$. Since $\varphi^{*}$ is negative the Defender has to turn right. The corresponding optimal trajectories are shown in Fig. 6(b).

\section{CONCLUSIONS}

The three agents differential game involving an Attacker, a Target, and a Defender, where the Defender has a bounded heading rate is solved. The restriction imposed on the Defender's turning rate is of great relevance since the Defender may be fired with an initial heading that is significantly different than the otherwise optimal heading when simple motion is assumed. The solution of this three agent differential game was illustrated with simulation results.

\section{APPENDIX}

\section{A. Proof of Theorem 1}

The distance between $D_{0}$ and $A$ is

$$
\begin{aligned}
\overline{A D_{0}} & =\sqrt{x^{2}+y^{2}} \\
& =\sqrt{x_{l}^{2}+r^{2}+L^{2}+2 x_{l}(r \sin \psi-L \cos \psi)} .
\end{aligned}
$$

The distance $d_{A}(\varphi)$ can be obtained as follows:

$$
d_{A}(\varphi)=\overline{A D_{0}} \frac{\sin \left(\psi-\lambda_{D}\right)}{\sin \left(2 \psi-2 \lambda_{D}\right)}=\frac{1}{2} \frac{\overline{A D_{0}}}{\cos \left(\psi-\lambda_{D}\right)} .
$$

The following expression determines the LOS angle from point $D_{0}$ to point $A$ in terms of the angle $\varphi$

$$
\lambda_{D}=\arctan \left(-\frac{\cos \psi+\varphi \sin \psi}{b+\sin \psi-\varphi \cos \psi}\right) .
$$

Further, the term $\cos \left(\psi-\lambda_{D}\right)$ can be written as follows

$$
\cos \left(\psi-\lambda_{D}\right)=\cos \psi \cos \lambda_{D}+\sin \psi \sin \lambda_{D}
$$

where

$$
\begin{aligned}
& \cos \lambda_{D}=\left(1+\frac{(\cos \psi+\varphi \sin \psi)^{2}}{(b+\sin \psi-\varphi \cos \psi)^{2}}\right)^{-\frac{1}{2}} \\
& \sin \lambda_{D}=\frac{-\frac{\cos \psi+\varphi \sin \psi}{b+\sin \psi-\varphi \cos \psi}}{\sqrt{1+\frac{(\cos \psi+\varphi \sin \psi)^{2}}{(b+\sin \psi-\varphi \cos \psi)^{2}}}} .
\end{aligned}
$$

By substitution of (26), (27), and (28) into (24) and simplification of the resulting expression we obtain (18).
From Fig. 4, we can see that $\chi(\varphi)=\psi-2 \lambda_{D}$. We obtain the coordinates of the interception point $I$ in terms of the variable $\varphi$ by using the expressions

$$
x_{I}(\varphi)=-d_{A} \cos \chi \quad y_{I}(\varphi)=d_{A} \sin \chi .
$$

The terms $\cos \chi$ and $\sin \chi$ can be written as follows

$$
\begin{aligned}
& \cos \left(\psi-2 \lambda_{D}\right)=\cos \psi \cos 2 \lambda_{D}+\sin \psi \sin 2 \lambda_{D} \\
& \sin \left(\psi-2 \lambda_{D}\right)=\sin \psi \cos 2 \lambda_{D}-\cos \psi \sin 2 \lambda_{D}
\end{aligned}
$$

where

$$
\cos 2 \lambda_{D}=\frac{1-\tan ^{2} \lambda_{D}}{1+\tan ^{2} \lambda_{D}} \quad \sin 2 \lambda_{D}=\frac{2 \tan \lambda_{D}}{1+\tan ^{2} \lambda_{D}} .
$$

Substituting (30)-(31) into (29) yeilds (20) and (21).

\section{REFERENCES}

[1] S. A. Ganebny, S. S. Kumkov, S. Le Ménec, and V. S. Patsko, "Model problem in a line with two pursuers and one evader," Dynamic Games and Applications, vol. 2, no. 2, pp. 228-257, 2012.

[2] H. Huang, W. Zhang, J. Ding, D. M. Stipanovic, and C. J. Tomlin, "Guaranteed decentralized pursuit-evasion in the plane with multiple pursuers," in IEEE Conference on Decision and Control and European Control Conference, 2011.

[3] R. L. Boyell, "Defending a moving target against missile or torpedo attack," IEEE Transactions on Aerospace and Electronic Systems, vol. AES-12, no. 4, pp. 522-526, 1976.

[4] R. L. Boyell, "Counterweapon aiming for defence of a moving target," IEEE Transactions on Aerospace and Electronic Systems, vol. AES16, no. 3, pp. 402-408, 1980.

[5] A. Ratnoo and T. Shima, "Line-of-sight interceptor guidance for defending an aircraft," AIAA Journal of Guidance, Control, and Dynamics, vol. 34, no. 2, pp. 522-532, 2011.

[6] T. Yamasaki and S. N. Balakrishnan, "Triangle intercept guidance for aerial defense," in AIAA Guidance, Navigation, and Control Conference, 2010.

[7] T. Yamasaki, S. N. Balakrishnan, and H. Takano, "Modified command to line-of-sight intercept guidance for aircraft defense," AIAA Journal of Guidance, Control, and Dyn., vol. 36, no. 3, pp. 898-902, 2013.

[8] A. Perelman, T. Shima, and I. Rusnak, "Cooperative differential games strategies for active aircraft protection from a homing missile," AIAA Journal of Guidance, Control, and Dynamics, vol. 34, no. 3, pp. 761773, 2011.

[9] I. Rusnak, H. Weiss, and G. Hexner, "Guidance laws in targetmissile-defender scenario with an aggressive defender," in IFAC World Congress, 2011.

[10] A. Ratnoo and T. Shima, "Guidance strategies against defended aerial targets," AIAA Journal of Guidance, Control, and Dynamics, vol. 35, no. 4, pp. 1059-1068, 2012.

[11] T. Shima, "Optimal cooperative pursuit and evasion strategies against a homing missile," AIAA Journal of Guidance, Control, and Dynamics, vol. 34, no. 2, pp. 414-425, 2011.

[12] V. Shaferman and T. Shima, "Cooperative multiple-model adaptive guidance for an aircraft defending missile," AIAA Journal of Guidance, Control, and Dynamics, vol. 33, no. 6, pp. 1801-1813, 2010.

[13] M. Pachter, E. Garcia, and D. W. Casbeer, "Active target defense differential game," in Annual Allerton Conference on Communication, Control, and Computing, 2014, pp. 46-53.

[14] E. Garcia, D. W. Casbeer, and M. Pachter, "Active target defense differential game with a fast defender," in American Control Conference, 2015, pp. 3752-3757.

[15] E. Garcia, D. W. Casbeer, and M. Pachter, "Cooperative strategies for optimal aircraft defense from an attacking missile," AIAA Journal of Guidance, Control, and Dyn., vol. 38, no. 8, pp. 1510-1520, 2015.

[16] J. D. Lawrence, A Catalog of Special Plane Curves. New York, Dover, 1972, pp. 190-191.

[17] B. O. Shubert, "A sequential method seeking the global maximum of a function," SIAM Journal on Numerical Analysis, vol. 9, no. 3, pp. 379-388, 1972.

[18] R. L. Springfield, "A computerized algorithm for sequential search of the global maximum." M.S. Thesis Department of Operational Analysis, Naval Postgraduate School Monterey, CA, Tech. Rep., 1970. 